\title{
mRCC: Erstlinientherapie entscheidend für Behandlungsverlauf
}

- Aktuell stehen mehrere zielgerichtete Therapien in der Erstlinienbehandlung des metastasierten Nierenzellkarzinoms (mRCC) zur Verfügung, wie der MultityrosinkinaseInhibitor Sunitinib (Sutent ${ }^{\oplus}$ ) und der mTORInhibitor Temsirolimus (Torisel ${ }^{\circledR}$ ). Diese Vielfalt an Optionen wirft die Frage auf, worauf

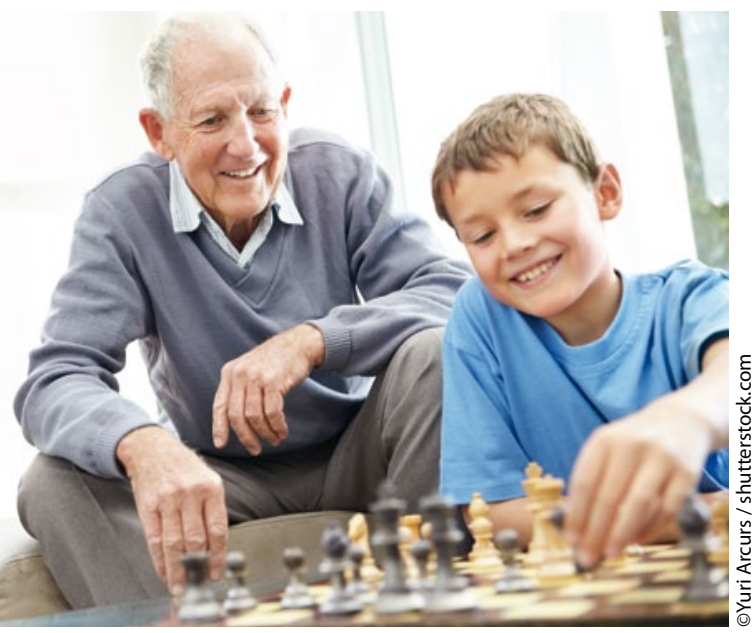

Das Ansprechen auf die Behandlung kann dazu beitragen, die Compliance der Patienten zu erhöhen. es heute in der Erstlinientherapie des mRCC ankommt. Eine Antwort darauf gab PD Dr. Michael Staehler, München, beim diesjährigen Deutschen Krebskongress (DKK) in Berlin: „Die Entscheidung, welches Medikament in der Erstlinientherapie zum Einsatz kommt, richtet sich maßgeblich nach der Effektivität.,Was wir am Anfang nicht erreicht haben, können wir später nicht mehr einholen." Es gelte, die Therapie früh zu beginnen und so lange wie möglich fortzusetzen. Wichtiger Wirksamkeitsparameter eines Medikaments sei dabei die Ansprechrate.

Mit einer objektiven Ansprechrate (ORR) von $47 \%$ und einem medianen Gesamtüberleben (OS) von 26,4 Monaten ist Sunitinib Referenzstandard in der mRCC-Erstlinientherapie [Motzer RJ et al. J Clin Oncol. 2009]. Bei mRCC-Hochrisikopatienten ist Temsirolimus als einzige zielgerichtete Substanz, die bei dieser Patientengruppe einen signifikanten Überlebensvorteil zeigen konnte, Erstlinienstandard [u.a. Miller K et al. Aktuelle Urol. 2011].

Die Wirksamkeitsdaten von Sunitinib und Temsirolimus unterstreichen, warum sie Referenzstandards in der Erstlinienbehandlung des mRCC sind. So betrug die Ansprechrate in der Sunitinib-Zulassungsstudie zur mRCCErstlinientherapie 47\% im Vergleich zu 12\% unter Interferon- $a$ (IFN- $\alpha ; p<0,001)$ [Motzer RJ et al. J Clin Oncol. 2009] Das Gesamtüberleben unter Sunitinib lag bei 26,4 Monaten (vs. 21,8 Monate unter IFN-a) und das progressionsfreie Überleben konnte mehr als verdoppelt werden ( 11 vs. 5 Monate). Unter Temsirolimus verlängerte sich das Gesamtüberleben bei Hochrisikopatienten in der Phase-III-Zulassungsstudie zur Erstlinienbehandlung signifikant auf 10,9 Monate (vs. 7,3 Monate unter IFN- $a$ ) [Hudes $\mathrm{G}$ et al. N Engl J Med 2007].

„Im Behandlungsalltag ist es besonders relevant, in der Erstlinie Tumorremissionen zu erzielen", unterstrich Staehler. Dementsprechend sei das Tumoransprechen ein aussagekräftiger, messbarer Wirksamkeitsparameter einer Therapie. Zudem könne eine Verkleinerung der Tumoren wesentlich dazu beitragen, assoziierte Beschwerden zu mildern und die Compliance der Patienten zu erhöhen.

Nach Informationen von Pfizer, Berlin

\section{Degarelix reduziert Prostatavolumen und LUTS}

— Bei Patienten mit lokalisiertem Hochrisikokarzinom oder lokal fortgeschrittenem Prostatakarzinom (PCa) kann eine Androgendeprivationstherapie (ADT) vor einer Strahlentherapie im Vergleich zur alleinigen Strahlentherapie die krankheitsspezifische Mortalität und das Gesamtüberleben verbessern [Payne et al. 2012]. Ob die Wirkung von GnRH-Blockern mit LHRH-Agonisten plus Antiandrogen in einem neoadjuvanten Therapiesetting vergleichbar ist, wurde bisher noch nicht analysiert. Auf dem diesjährigen EAU-Kongress wurden nun die Daten von zwei aktuellen Phase-IIllb-Studien (CS30 und CS31) präsentiert.

In der CS30-Studie wurden die Effekte des GnRH-Blockers Degarelix (Firmagon ${ }^{\circledR}$ ) als neoadjuvante Hormontherapie (HT) vor der Strahlentherapie bei PCa-Patienten mit mittlerem bis hohem Risiko im Vergleich zum LHRH-Agonisten Goserelin mit Bicalutamid untersucht. Die in die Studie eingeschlos- senen Patienten wurden 3:1 auf Degarelix (240/80 mg; $n=181$ ) oder Goserelin (3,6 mg; $\mathrm{n}=65)$ mit Bicalutamid randomisiert.

Unter Degarelix schrumpfte bis Woche 12 das mittlere prozentuale Prostatavolumen um 36\%, unter Goserelin mit Bicalutamid um $35 \%$. Degarelix verbesserte verglichen mit Goserelin mit Bicalutamid neoadjuvant bei Patienten mit einem Ausgangswert von 8-19 Punkten im IPSS (International Prostate Symptom Score) die Symptome des unteren Harntraktes (LUTS) signifikant. Unter dem GnRH-Blocker sank der IPSS um 2,99 Punkte $(\mathrm{n}=72)$, unter Goserelin plus Bicalutamid um 0,48 Punkte $(n=23)$. Degarelix könnte demzufolge per se bessere Antitumoreffekte haben. Möglicherweise ist auch die unterschiedliche Wirkung des Blockers und des Agonisten auf die peripheren GnRH-Rezeptoren in der Prostata und der Harnblase hierfür verantwortlich. In beiden Studien-Armen wurde eine ähnliche Gesamtinzidenz an un- erwünschten Ereignissen beobachtet; die am häufigsten berichteten unerwünschten $\mathrm{Ne}$ benwirkungen waren die üblichen erwarteten Ereignisse der Androgendeprivationstherapie.

Die CS31-Studie untersuchte die Reduktion des Prostatavolumens unter Degarelix versus Goserelin plus Bicalutamid bei einem breiten PCa-Patientenspektrum. Degarelix war hinsichtlich der Schrumpfung des Prostatavolumens der Vergleichsmedikation nicht unterlegen, so die Ergebnisse in Woche 12. Bei symptomatischen Patienten wurden die LUTS unter dem GnRH-Blocker signifikant stärker verbessert als unter dem LHRH-Agonisten mit Bicalutamid.

Nach Informationen von

Ferring, Kiel 